\title{
Simulating Systems-of-Systems Dynamic Architectures
}

\author{
Wallace Manzano ${ }^{1}$, Valdemar Vicente Graciano Neto $^{2}$, Elisa Yumi Nagakawa ${ }^{1}$ \\ ${ }^{1}$ Instituto de Ciências Matemáticas e de Computação \\ Universidade de São Paulo (USP) - São Carlos - SP - Brazil \\ ${ }^{2}$ Instituto de Informática \\ Universidade Federal de Goiás (UFG) - Goiânia - GO - Brazil \\ wallace.manzano@usp.br, valdemarneto@inf.ufg.br, elisa@icmc.usp.br
}

\begin{abstract}
Systems-of-Systems (SoS) combine heterogeneous, independent systems to offer complex functionalities for highly dynamic smart applications. Due to their critical nature, SoS should be reliable and work without interruption that could cause serious losses. SoS architectural design can facilitate the prediction of the impact of failures due to SoS behavior. However, existing approaches do not support such evaluation. The main contribution of this paper is to present Dynamic-SoS, an approach to predict, at design time, the SoS architectural behavior at runtime to evaluate whether the SoS can sustain their operation. Results of our multiple case studies reveal Dynamic-SoS is a promising approach that could contribute to the quality of SoS by reliably enabling prior assessment of their dynamic architecture.
\end{abstract}

\section{Introduction and Background}

Context. Software-intensive systems have been increasingly required to interoperate amongst themselves, communicating, exchanging, and using information exchanged ${ }^{1}$. Consequently, a distinct class of systems known as Systems-of-Systems (SoS $)^{2}$ has arisen. An SoS results from operationally and managerially independent software-intensive systems (called constituents) working together to fulfill complex missions (Maier 1998). SoS are often linked to critical domains, such as smart traffic control systems, crisis response management, and national defense systems.

Due to their inherent criticality, it is imperative to SoS be reliable, that is, their operation must be correct and happen within what is expected, without causing harm or threatening people who use their services. To guarantee a reliable operation for SoS, it is important to establish strategies to maintain an SoS operation in progress, despite a high degree of dynamism, with new constituents joining the SoS at runtime, others leaving it, or being replaced (Heegaard and Schoitsch 2015). In addition, given the criticality of the processes they automate, it is essential that their operation is not interrupted and that any problems in one or more of their constituents are automatically solved by automatic and dynamic reconfiguration of their architecture at runtime to assess the feasibility of their continuous operation.

Simulation techniques have been successfully used, especially in software engineering (de França and Travassos 2016), to support the visualization of the system's

\footnotetext{
${ }^{1}$ http: / / www.himss.org/library/interoperability-standards / what-is-interoperability

${ }^{2}$ For sake of simplicity, the acronym SoS is used interchangeably to express singular and plural.
} 
dynamic behaviors. Simulations can anticipate, at design time, failures and behaviors that can potentially occur at runtime. In particular, the simulation of software architectures can be achieved by using simulation models with dynamic reconfiguration to predict architectural configuration changes at runtime for single systems (Bogado et al. 2017). Indeed, Dynamic Structure Discrete Event System Specification (DS-DEVS) (Zeigler et al. 2012) is one of the most used simulation formalisms for modeling behavior of systems from input, output, and their states, as well as the coupling of these systems, adopted by Bogado et al. (2017).

Problem. Despite the suitability of simulation formalisms to predict behaviors with dynamic reconfiguration support, a complex task arises from guaranteeing precision in simulating SoS software architectures, i.e., simulating the behavior of multiple constituent systems and their relationship to each other, and to the environment at runtime while still considering the dynamic reconfiguration of their constituent systems. Existing simulation formalisms have limitations on the precise representation of SoS software architectures, including the representation of different types of systems, environment representation, and dynamic reconfiguration. Despite the formalism supporting the dynamic architecture representation, the mechanism to implement it is arbitrarily chosen by the user. On the other hand, pure adoption of architectural description languages (ADL) also suffers from limitations on the precise description of dynamism of an architecture composed by multiple systems and the impact of it on the provided behavior, as recognized by Guessi et al. (2015). The main research question addressed in this work is: How to provide a means to assure that the SoS operation is trustworthy, i.e., will it be maintained on-the-fly, despite the inherent SoS dynamic architecture?

Solution. To address this question, the aim of this paper is to present DynamicSoS, an approach established by the undergraduate student for simulating SoS software architectures with dynamic reconfiguration support. Our approach uses a SosADL to DEVS model transformation (SosADL2DEVS) that automatically generates simulation models from SoS software architecture specifications. The main contributions of Dynamic-SoS were: (i) the proposition of a set of canonical dynamic reconfiguration operators for SoS software architectures; (ii) the extension of an existing model transformation by adding a dynamic reconfiguration controller (DRC) structure, which enables simulation models in DEVS to show and manage dynamic reconfigurations at runtime; (iii) the engineering of DRC itself, since such an idea could be used for other simulation formalisms, as well; and (iv) the characterization of the process to assess SoS behaviors considering the associated dynamic architecture. We evaluated our approach through of multiple case studies in three distinct domains: Flood Monitoring SoS, Smart Building SoS, and Space SoS. From a tringulation of the results obtained from these case studies, we concluded our approach successfully supports simulation of SoS while accounting for dynamic architectures. As a result, our work may potentially be used to evaluate SoS dynamic architectures, enhancing SoS quality by enabling the visualization of possible problems that the SoS may exhibit besides predicting/anticipating SoS architectural behavior at runtime, thus evaluating whether the SoS can sustain their operation.

This paper is organized as follows: Section 2 presents Dynamic-SoS, Section 3 
presents our evaluation and results, Section 4 summarizes the contributions of this work, and Section 5 contains final remarks and directions for future work.

\section{Dynamic-SoS Approach}

Dynamic-SoS is a model-based approach to automatically create SoS simulations with dynamic reconfiguration. To support the dynamic reconfiguration, we established a set of dynamic reconfiguration operations based on the canonical set of changes initially proposed by Cavalcante et al. (2015). Cavalcante et al. observed single systems may have their architectures modified at runtime due to the creation of a new component, removal of a component, attachment of a component (attachment), and detachment of a component. For SoS software architectures, we reviewed such operators and defined four canonical operators to achieve dynamics in such architectures. In our approach, dynamic reconfiguration in an SoS software architecture is based on constituent addition, constituent deletion, constituent replacement, and architecture reorganization. Our approach uses a SosADL to DEVS model transformation (SosADL2DEVS) that automatically generates simulation models from SoS software architecture specifications,

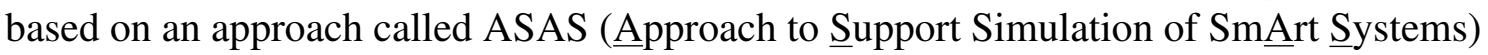
(Graciano Neto et al. 2018a). Dynamic-SoS was established by adding the generation and monitoring of dynamic architectures for that SosADL2DEVS transformation produced by Graciano Neto et al. (2018a), as depicted in Figure 1. Moreover, we established a set of activities to systematize the use of Dynamic-SoS approach, as follows.

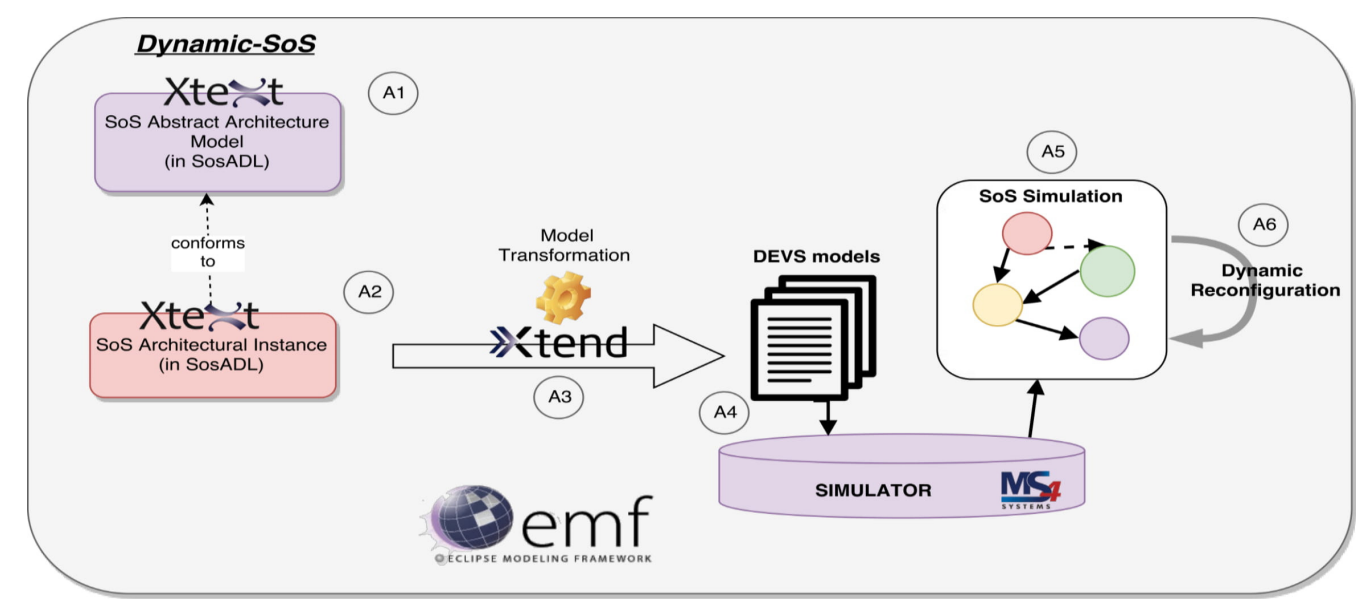

Fig. 1. Dynamic-SoS approach.

Activity 1 (A1). SoS architectures are specified by means of coalitions, which express the policies and define bindings among constituents. A coalition may involve many constituents, or exactly one constituent and multiple mediators. An SoS software architecture results from the selection, at runtime, of possible constituent systems that may participate in the SoS. To generate the initial architectural arrangement for the simulation, we use a method defined by Guessi et al. (2016);

Activity 2 (A2). Execution of the model transformation on the concrete model (in SosADL) to automatically produce a DEVS simulation code with support for the dynamic reconfiguration of an SoS architecture;

Activity 3 (A3). Deployment, i.e., the process of managing files of the atomic and coupled models obtained as an outcome of the transformation, deploying them in the specific 
packages/directories of the project to be simulated in $\mathrm{MS}_{4} \mathrm{ME}^{3}$ (the simulation environment);

Activity 4 (A4). Simulation execution that consists of launching the simulation; and Activity 5 (A5). Monitoring architectural reconfiguration using the dynamic reconfiguration operators, comprising the observation of the SoS dynamics at runtime, and registering the execution traces in log files for posterior analysis.

\subsection{Dynamic reconfiguration controller}

Dynamic-SoS is a programmed exogenous reconfiguration approach, which means that an entity termed as Dynamic Reconfiguration Controller (DRC) is responsible for managing every architectural change that occurs in the whole structure. DRC is an artificial architectural element that owns control over all elements of the software architecture and manages the architectural changes. DRC is added to the simulation to support the user in conducting architectural changes at runtime through the dynamic reconfiguration operators. Figure 2 presents the state machine of DRC. From the DEVS simulation model perspective, DRC is an atomic model, whose function is to execute the four dynamic reconfiguration operators while maintaining properties of the initial architecture configuration.

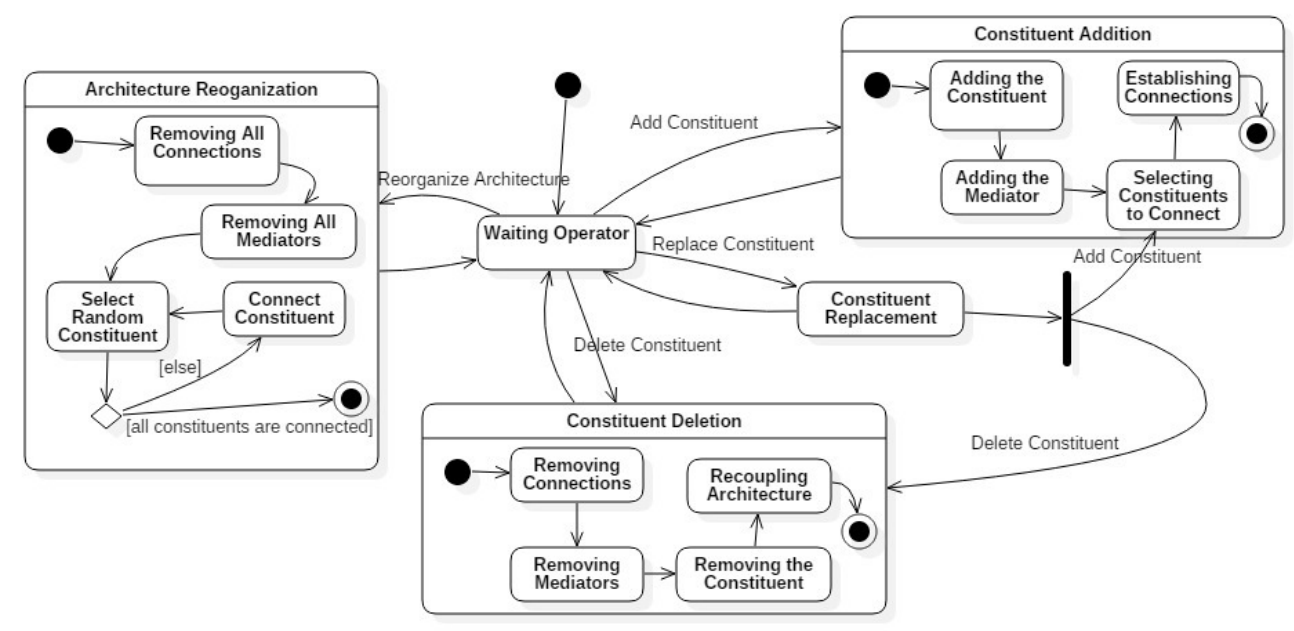

Fig. 2. State machine implemented in DEVS to materialize the four canonical reconfiguration operators performed by the Dynamic Reconfiguration Controller.

To execute a reconfiguration operator, it is necessary to send a signal to the DRC with the operator to be performed and, if the operator is not a reorganization, the constituent in which the operator will be executed (in the addition the type of the constituent to be added) is also required.

\subsection{Adding Support of Dynamic Reconfiguration through a Model Transformation}

All SosADL elements are mapped to DEVS to create a functional simulation. Transformation rules automatically create DRC from the concrete SosADL architecture and add

\footnotetext{
${ }^{3}$ http://www.ms4systems.com/pages/ms4me.php
} 
it in the simulation model. This controller holds and makes available to the user the four dynamic reconfiguration operators.

The model transformation generates three main elements related to the dynamic architecture of the target simulation model, which are:

- DRC: As presented in Section 2.1, it consists of an atomic model that manages all changes in the simulation, as shown in Figure 2. For that, it manages connections and mediators among constituents, so that the new arrangements remain consistent with the original architecture;

- Identification flags: In DEVS, there is no distinction between constituent systems and mediators, all of which are transformed into atomic models. However, this differentiation is necessary to maintain the functional architecture after running a dynamic reconfiguration operator. To artificially bring that to DEVS, we added two identification flags to the atomic models: one to check if the system is a mediator or not (a binary variable), and another that is the constituent type name, such as sensor, transmitter, or gateway; and

- Connections of all constituents with the DRC in the coupled model: This is necessary to enable the controller to communicate with all constituents and to remove some of them, if necessary.

\section{Evaluation}

Dynamic-SoS was evaluated through of nine case studies, three of which were designed specifically for the evaluation of the approach, and the other six designed for the evaluation of other works. Dynamic-SoS was able to simulate 395 constituents in a single simulation and successfully ran 133 hours of simulation (Table 1). Due to the large number of constituents and the long duration of the simulations, auxiliary mechanisms for evaluation were implemented, such as a log for human experts, and automatic procedures for measuring and delivering results regarding the delivery percentage of the behaviors.

Three case studies designed for Dynamic-SoS evaluation were conducted in three distinct domains (Manzano et al. 2019):

1. Flood Monitoring SoS (FMSoS): a SoS with no central authority and being part of a smart city responsible for monitoring rivers that cross urban areas and notifying the population about potential floods that can quickly occur, causing huge damage and risk for population. FMSoS is deployed along the river and its sensors are spread on the riverbank's edges and data are transmitted to a gateway. Additionally, drones fly over the river and return to their bases to recharge and communicate flood threat alerts. In parallel, people walking close to the river can also contribute by communicating water level increases through a crowdsensing mechanism supported by mobile apps;

2. Space SoS: a real system called Environmental Data Collection System (SBCDA, in Portuguese). Space SoS is operated and managed by the Brazilian National Institute for Space Research (INPE, in Portuguese), which provided us real data and know-how to precisely model the entire system. Space SoS is responsible to perform two concurrent missions (environmental data collection and image capture), and is composed of satellites, ground station, command and control center, 
and data collection platforms, which each of then can be managed by different institutions; and

3. Smart Building: a SoS that provides important services to their residents and visitors, such as energy savings, and light control by sensors, and fire alarms in case they happen. Dynamic-SoS approach was also evaluated using a Smart Building SoS (SBS), which is composed of three other SoS: fire system (composed of sensors, alarms, and sprinklers), lighting system (composed of sensors and lamps), and room (composed of sensors, air conditioners, and alarms). In addition, SBS has a Smart Building Control Unity (SBCU), which is responsible by managing constituent systems of the building.

In this work, the matter of interest is the simulation of SoS dynamic architectures. We intended to observe whether Dynamic-SoS was well-succeeded for both: (i) automatically providing simulation models that support dynamic reconfigurations; and (ii) supporting the analysis of the impact of such reconfigurations on the SoS architecture, in particular, in regards to the sustainability of SoS emergent behaviors.

We executed three different evaluations, involving FMSoS, Smart Building, and Space SoS. For all of them, we applied the same set of architectural changes in the same order, and observed their impact on the data transmission rates (intimately aligned with the emergent behaviors provided by the SoS) expressed by the percentage of data that was successfully received in a gateway or that passed by an specific constituent in regards to the total number of data that was provided as stimuli to feed each simulation.

Figure 3 shows data collected from simulations. This data presents the percentage of collected data during the case studies (y axis) and performed operators (color, as specified in the legend). This data supports us to analyze the SoS availability and performance to fulfill its missions during its execution and predict how changes in the architecture of SoS will impact its performance, allowing to anticipate eventual problems.

Additionally, we can analyze the impact of executing operators in the architecture. Figure 4 shows the impact of the addition operator on receiving data (y axis) and shows in which constituent type was performed the operator (point color). This chart allows us to analyze how the increase in the number of constituents affects data transmission. In all case studies, the addition of sensors/collectors decreased the data received, while addition of constituents that receive these data increases the rate of data collection. This helps us to draw a trade-off on the acquisition of new constituents, making it possible, for instance, to conclude that adding a second gateway in the FMSoS increases the data reception rate by $20 \%$, which is the same gain that happens when adding a second satellite to the Space SoS.

Then, after analyzing and triangulating results obtained from three different independent studies, we can answer the raised research question for the case studies: Are the architectural changes successful, giving rise to new SoS coalitions in a valid operational state? The answer is Yes. In 100\% of the cases in the three studies, Dynamic-SoS was successful to support dynamic reconfigurations in SoS software architectures, leading the architecture to valid operational states in all obtained coalitions.

Besides the three case studies designed for the Dynamic-SoS evaluation, our approach supported the execution of other six cases, totaling nine case studies. Table 1 


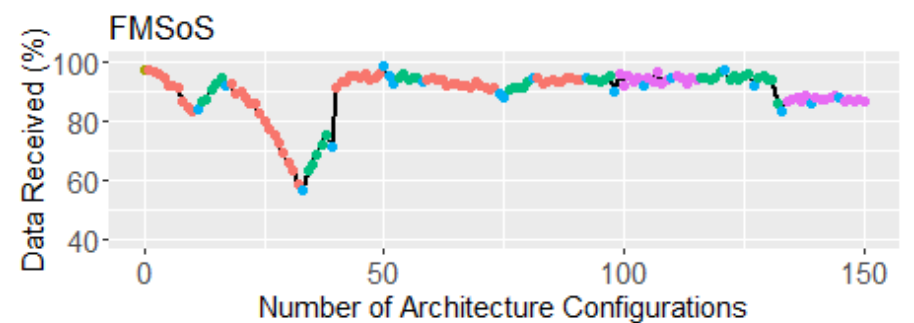

Dynamic

Reconfiguration

Operator

- Addition

- None

- Removal

- Reorganization

- Replacement

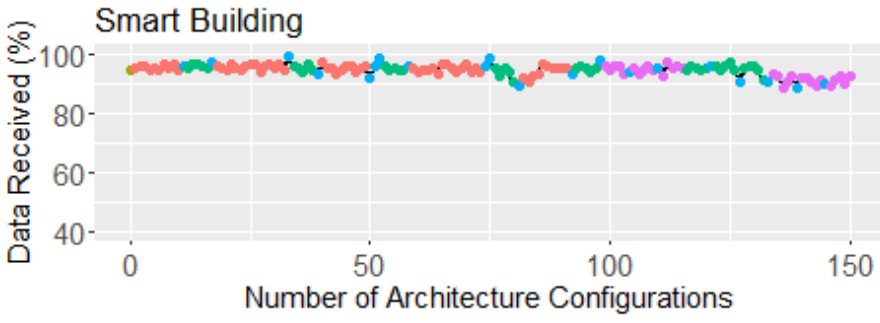

Dynamic

Reconfiguration

Operator

- Addition

- None

- Removal

- Reorganization

- Replacement

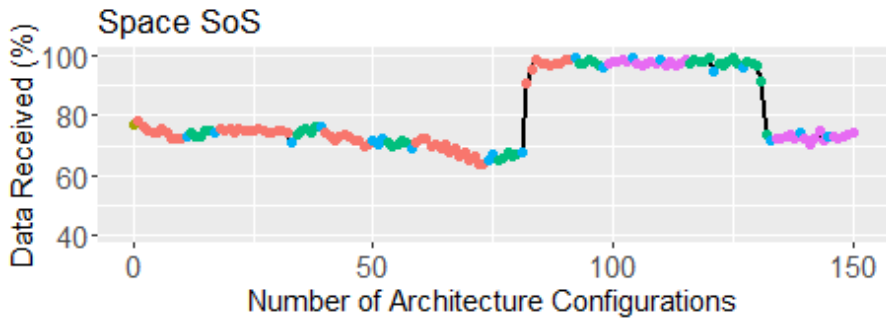

Dynamic

Reconfiguration

Operator

- Addition

- None

- Removal

- Reorganization

- Replacement

Fig. 3. Chart showing the rate of receipt of data throughout the case studies.

shows the studies that used Dynamic-SoS, their domain, number of lines of code (LOC) of the models specified in SosADL, amount generated in DEVS by model transformation, maximum number of constituent reached in the case study, and total simulation time. With these results, we were able to evaluate that Dynamic-SoS was successful in generating more than 1 million LOC, and successfully simulated more than 130 hours.

Tab. 1. Lines of code generated in each case stury.

\begin{tabular}{|l|l|l|l|l|l|l|}
\hline$\#$ & Study & Domain & $\begin{array}{l}\text { SosADL } \\
\text { (LOC) }\end{array}$ & $\begin{array}{l}\text { DEVS } \\
\text { (LOC) }\end{array}$ & $\begin{array}{l}\text { Maximum } \\
\text { Number of } \\
\text { Constituents }\end{array}$ & $\begin{array}{l}\text { Total simu- } \\
\text { lation time } \\
\text { (hours) }\end{array}$ \\
\hline 1 & (Graciano Neto et al. 2018a) & FMSoS & 701 & 8,563 & 70 & 6.33 \\
\hline 2 & (Manzano et al. 2019) & FMSoS & 701 & 8,563 & 52 & 5.33 \\
\hline 3 & (Graciano Neto et al. 2017) & FMSoS & 1,154 & 56,185 & 112 & 6.2 \\
\hline 4 & (Graciano Neto et al. 2018b) & FMSoS & 660 & 3,332 & 89 & 2.6 \\
\hline 5 & (Manzano et al. 2019) & Smart Building & 6,724 & 219,930 & 395 & 9.66 \\
\hline 6 & (Graciano Neto et al. 2018c) & Space SoS & 640 & 11,195 & 4 & 0.72 \\
\hline 7 & (Graciano Neto et al. 2019) & Space SoS & 2,399 & 128,884 & 311 & 27 \\
\hline 8 & (Manzano et al. 2019) & Space SoS & 2,677 & 378,557 & 155 & 21.5 \\
\hline 9 & (Graciano Neto et al. 2019) & Space SoS & 4,014 & 497,040 & 258 & 34 \\
\hline
\end{tabular}

\section{Contributions}

Publications. This work resulted in two publications:

1. Manzano, W., Graciano Neto, V. V., and Nakagawa, E. Y. (2019). Dynamic-SoS: An approach for the simulation of system-of-systems dynamic architectures. The Computer Journal (Qualis A2), 62:1 - 23. 

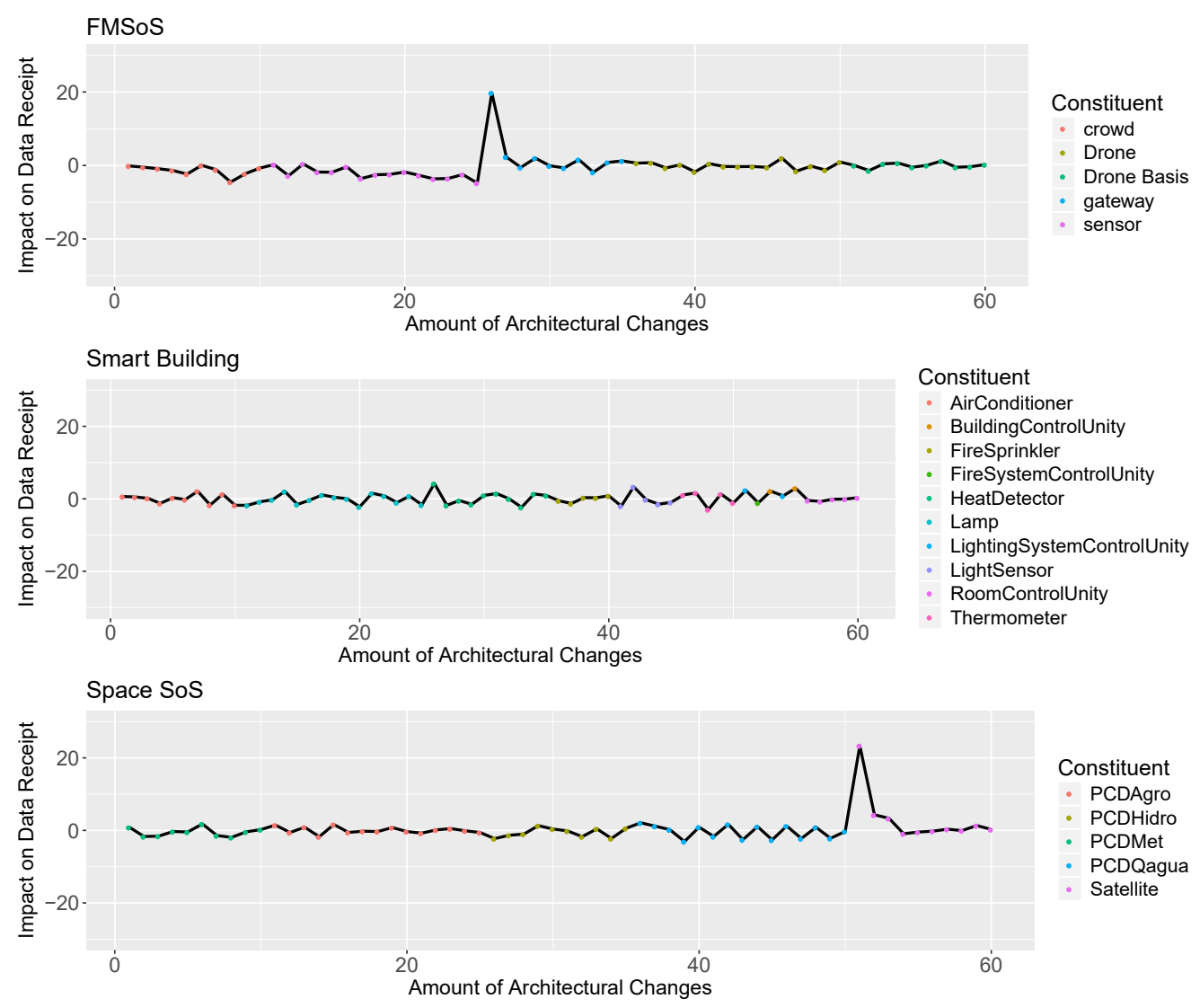

Fig. 4. Chart presenting the impact of the addition of a constituent in the simulation.

2. Manzano, W., Graciano Neto, V. V., and Nakagawa, E. Y. (2018). Verificação Estatística de Modelos de Arquiteturas de Software de Sistemas-de-Sistemas. In 26o Simpósio Internacional de Iniciação Científica da USP (SIICUSP)

3. Manzano, W., Graciano Neto, V. V., and Nakagawa, E. Y. (2017). Simulação de Arquiteturas de Software de Sistemas-de-Sistemas com Suporte à Reconfiguração Dinâmica. In 25o Simpósio Internacional de Iniciação Científica da USP (SIICUSP)

In addition to these two self-authored important publications, other several publications were achieved using results provided by the approach developed by this student and having him as a co-author, including one journal publication Qualis B1 (Graciano Neto et al. 2017), two full papers in premier international conferences Qualis A1 (Graciano Neto et al. 2018c; Graciano Neto et al. 2018a), and also other important four conferences and workshop papers ${ }^{4}$.

Impact. With regard to this work, important contributions were brought:

1. Characterization of SoS dynamic architectures operators. Providing dynamic architectures inherently depends on designing each possible change that can be performed on these architectures. Those changes should be well-defined and the final architecture should always deliver a valid operational state. Dynamic

\footnotetext{
${ }^{4}$ The list can be checked herein: https ://dblp.org/pers/hd/m/Manzano:Wallace
} 
reconfiguration operators were previously established for single systems software architectures. However, when considering multiple interoperable systems present in an SoS, there was a gap to be bridged. Dynamic-SoS builds a remarkable contribution on previous advances by providing a robust and canonical set of dynamic changes that can be adopted to represent any change that a SoS software architecture can suffer. We state this as a contribution that can be replicated in other contexts that require SoS dynamic architectures;

2. Generation of dynamic models from static models. Dynamic-SoS also provides a means to automatically derive SoS simulation models from static specification of SoS software architectures with a single initial coalition; and

3. A process to include dynamics in SoS software architecture models. By using a model transformation approach, we established a process that encompasses activities necessary to observe the dynamics of SoS via simulations, passing by the SoS software architecture specification step until reaching the assessment of different architectural configurations.

In addition, we extend Dynamic-SoS through a plugin to communicate with a statistical model checker to automatically perform a statistical verification of functional and architectural properties to automatically provide a level of trust in which the architecture is able to meet the properties. Besides, Dynamic-SoS can be expanded to cover other purposes of study, such as measuring other specific quality attributes, analyzing the threshold or limit of constituents that still maintain the SoS feasible, applying a set of random reconfigurations and studying the architecture behavior, and predicting the impact of specific constituents on the entire SoS architecture.

\section{Final Remarks}

SoS architectural design is a challenging task due to diverse architectural configurations a SoS can assume at runtime and the complexity of assessing the impact of such changes still at design time. Dynamic-SoS contributes to this scenario by providing an infrastructure that allows SoS architects to predict, via simulations automatically generated at design-time, the SoS dynamic architectures, besides visualizing the SoS dynamics, and their impact on the SoS associated behavior. We conducted nine case studies, generating simulation models and analyzing the impact of changes on the functionalities provided by the modeled SoS. Results brought evidences that Dynamic-SoS is a feasible and effective approach to predict SoS behavior associated to the inherent dynamics associated to their architecture. Motivated by these results, our future work involves dealing with real SoS dynamic architectures at runtime (i.e., after SoS deployment), advancing on models@runtime research branch. We intend to draw strategies for the synchronization between coalitions and SoS architectural specification, preserving SoS architectural consistency, avoiding degradation and assuring the quality of this class of systems that has been increasingly applied in several critical, complex application domains.

\section{Acknowledgments}

The authors thank São Paulo Research Foundation (FAPESP) (grants: 2014/02244-7, 2017/06195-9, 2017/17448-5, and 2018/21517-5). 


\section{References}

[Bogado et al. 2017] Bogado, V., Gonnet, S., and Leone, H. (2017). DEVS-based methodological framework for multi-quality attribute evaluation using software architectures. In CLEI, pages 1-10, Cordoba, Argentina.

[Cavalcante et al. 2015] Cavalcante, E., Batista, T. V., and Oquendo, F. (2015). Supporting dynamic software architectures: From architectural description to implementation. In WICSA, pages 31-40, Montreal, Canada.

[de França and Travassos 2016] de França, B. B. N. and Travassos, G. H. (2016). Experimentation with dynamic simulation models in software engineering: planning and reporting guidelines. Empirical Software Engineering, 21(3):1302-1345.

[Graciano Neto et al. 2017] Graciano Neto, V. V., Barros Paes, C. E., Garcés, L., Guessi, M., Manzano, W., Oquendo, F., and Nakagawa, E. Y. (2017). Stimuli-SoS: a modelbased approach to derive stimuli generators for simulations of systems-of-systems software architectures. Journal of the Brazilian Computer Society, 23(1):1-22.

[Graciano Neto et al. 2018a] Graciano Neto, V. V., Garcés, L., Guessi, M., Paes, C., Manzano, W., Oquendo, F., and Nakagawa, E. Y. (2018a). ASAS: An approach to support simulation of smart systems. In 51st HICSS, pages 5777-5786, Big Island, Hawaii, USA.

[Graciano Neto et al. 2018b] Graciano Neto, V. V., Manzano, W., Garcés, L., Guessi, M., Oliveira, B., Volpato, T., and Nakagawa, E. Y. (2018b). Back-SoS: Towards a modelbased approach to address architectural drift in systems-of-systems. In $S A C$, pages 1-3, Pau, France. ACM.

[Graciano Neto et al. 2018c] Graciano Neto, V. V., Manzano, W., Rohling, A. J., Ferreira, M. G. V., Volpato, T., and Nakagawa, E. Y. (2018c). Externalizing patterns for simulations in software engineering of systems-of-systems. In SAC.

[Graciano Neto et al. 2019] Graciano Neto, V. V., Paes, C. E., Rohling, A. J., Manzano, W., and Nakagawa, E. Y. (2019). Modeling \& simulation of software architectures of systems-of-systems : An industrial report on the brazilian space system. In SpringSim, pages 1-12. Accepted.

[Guessi et al. 2015] Guessi, M., Graciano Neto, V. V., Bianchi, T., Felizardo, K. R., Oquendo, F., and Nakagawa, E. Y. (2015). A systematic literature review on the description of software architectures for systems of systems. In SAC, pages 1433-1440, Salamanca, Spain.

[Guessi et al. 2016] Guessi, M., Oquendo, F., and Nakagawa, E. Y. (2016). Checking the architectural feasibility of systems-of-systems using formal descriptions. In SoSE, pages 1-6, Kongsberg, Norway.

[Heegaard and Schoitsch 2015] Heegaard, P. E. and Schoitsch, E. (2015). Introduction to the special theme - trustworthy systems of systems. ERCIM News, 2015(102):8-9.

[Maier 1998] Maier, M. W. (1998). Architecting principles for systems-of-systems. Systems Engineering, 1(4):267-284.

[Manzano et al. 2019] Manzano, W., Graciano Neto, V. V., and Nakagawa, E. Y. (2019). Dynamic-SoS: An Approach for the Simulation of Systems-of-Systems Dynamic Architectures. The Computer Journal, 62:1-23.

[Zeigler et al. 2012] Zeigler, B. P., Sarjoughian, H. S., Duboz, R., and Souli, J.-C. (2012). Guide to Modeling and Simulation of Systems of Systems. Springer, Berlim, Germany. 